\title{
Refractory Metastatic Insulinoma Treated with Everolimus, Complicated by Cryptogenic Organizing Pneumonia
}

\author{
Charles J. Kim', Devon McKenzie ${ }^{2}$, Joe K. Joseph ${ }^{1}$, Yasemin Aytaman ${ }^{1}$, \\ Milay Luis Lam ${ }^{3}$, Lina Soni ${ }^{3}$, Samy I. McFarlane, \\ ${ }^{1}$ Department of Medicine, State University of New York: Downstate Medical Center \\ ${ }^{2}$ Department of Hematology and Oncology, State University of New York: Downstate Medical Center \\ ${ }^{3}$ Department of Endocrinology, State University of New York: Downstate Medical Center \\ ${ }^{4}$ Department of Medicine and Department of Endocrinology, State University of New York: Downstate Medical Center \\ *Corresponding author: samy.mcfarlane@downstate.edu
}

Received April 26, 2019; Revised June 02, 2019; Accepted June 18, 2019

\begin{abstract}
Insulinoma is a rare neuroendocrine pancreatic islet cell tumor of which the majority are benign and solitary. Its estimated incidence is 2 to 4 cases per 1 million person-years. We report the case of an 82-year-old female who presented with metastatic insulinoma to the liver and retroperitoneal lymph nodes. It was diagnosed based on positive Whipple's triad, elevated insulin, elevated C-peptide, and negative insulin auto-antibody. Her disease was initially managed with diazoxide $100 \mathrm{mg}$ orally every 8 hours, octreotide LAR $30 \mathrm{mg}$ intramuscularly every month, and sunitinib $12.5 \mathrm{mg}$ orally three times a day. However, patient had recurrent symptoms and imaging consistent with worsened metastatic insulinoma; and thus, treatment was changed to everolimus $10 \mathrm{mg}$ PO daily. Over the subsequent 10 months, the patient developed progressive shortness of breath and hypoxemia with and oxygen saturation (SpO2) of $89 \%$ on room air. Computerized tomography (CT) and lung biopsy were consistent with cryptogenic organizing pneumonia (COP) temporally associated with the initiation of everolimus. She was started on prednisone $1 \mathrm{mg} / \mathrm{kg} /$ day and within 48 hours, her symptoms and hypoxemia improved to SpO2 of 98-99\% at room air and her repeat CT chest showed marked disease improvement. Given her good response with everolimus, it was continued in conjunction with the prednisone and to this day, patient has had a significant therapeutic response with normoglycemia and stable, well-controlled symptoms. This case is unique because to our knowledge, it is the first reported case of a patient with metastatic insulinoma complicated by the development of cryptogenic organizing pneumonia. It reaffirms the causal association between everolimus and cryptogenic organizing pneumonia that has been reported numerous times in literature. However, there has been no reported cases showing that the COP can be managed with prednisone concurrently with everolimus for the metastatic insulinoma without diminished clinical benefit. While pulmonary complications have been cited as reasons for discontinuation of everolimus therapy, our case report highlights the use of steroids as a viable therapeutic strategy that allows successful therapy with everolimus to be continued. In addition to presenting this case, we will also do a thorough review of the literature surrounding the available therapeutic options of metastatic insulinoma. This will include surgery, somatostatin analogs, antimicrobials, potassium channel activators, VEGF-A inhibitors, alkylating agents and mTOR inhibitors to provide a more in-depth picture of how we treat metastatic insulinoma.
\end{abstract}

Keywords: Insulinoma, cryptogenic organizing pneumonia, everolimus

Cite This Article: Charles J. Kim, Devon McKenzie, Joe K. Joseph, Yasemin Aytaman, Milay Luis Lam, Lina Soni, and Samy I. McFarlane, "Refractory Metastatic Insulinoma Treated with Everolimus, Complicated by Cryptogenic Organizing Pneumonia.” American Journal of Medical Case Reports, vol. 7, no. 7 (2019): 125-132. doi: 10.12691/ajmcr-7-7-2.

\section{Introduction}

Due to the rarity of insulinoma, other causes of symptomatic hypoglycemia associated with fasting must be explored before considering it as the diagnosis. This includes, but is not limited to, malnourishment, sepsis, drugs (e.g., quinolones, beta-blockers, alcohol,
ACE-inhibitors/ARB, insulin, insulin secretagogue), hormone deficiency (e.g., cortisol, glucagon, epinephrine), factitious hypoglycemia, auto-antibodies against insulin (e.g. Graves' disease, lupus, rheumatoid arthritis), liver failure, oncologic (e.g., rhabdomyosarcoma, leiomyosarcoma, and hemangiopericytoma), and renal causes (e.g., Fanconi syndrome).

To diagnose insulinoma with certainty, patients must present with some or all of the symptoms of Whipple's 
triad and the patient's serum insulin levels must be below normal. The Whipple's triad consists of episodic hypoglycemia (<50 mg/dL), symptoms of hypoglycemia (confusion, anxiety, paralysis, stupor, coma), and reversal of symptoms with glucose administration.

The rarity of insulinoma was described in a 60-year study by Service et. al of Mayo clinic that found 224 cases of a histologically-confirmed functioning insulinoma, approximately 2 to 4 cases per 1 million person-years. In this study median age at onset was 47 years and 59\% were women. Further characterizing this group, they found 7.6\% had multiple endocrine neoplasia (MEN-1) and $5.8 \%$ had malignant insulinoma. Additionally, the survival of malignant insulinoma was worse compared to the study cohort without malignancy ( $29 \%$ vs $88 \%$, respectively). Patient survival differed based on the patients' older age, MEN-1 mutation, those diagnosed prior to surgical innovations, and treatment modalities [1].

This report focuses on different medical treatment methods and complications associated with everolimus in the treatment of a patient with refractory metastatic insulinoma.

\section{Case Report}

We present the case of an 82-year-old woman with a past medical history significant only for hypertension. Her initial presentation to our institution was 10 years ago when she presented with the chief complaint of two syncopal episodes over two weeks, associated with diaphoresis and malaise. There was no reported seizure activity, urinary incontinence, tongue biting, chest pain, palpitations or shortness of breath. She had serum glucose of $38 \mathrm{mg} / \mathrm{dL}$ and hypokalemia of $3.3 \mathrm{mEq} / \mathrm{L}$, for which she was emergently managed with intravenous potassium chloride and dextrose. 72-hour fast was performed and when her glucose was $<45 \mathrm{mg} / \mathrm{dL}$, the fast was stopped and serum cortisol $=18$ $\mathrm{mcg} / \mathrm{dL}$, insulin= $18.77 \mathrm{mcU} / \mathrm{mL}, \mathrm{C}$-peptide= $3.85 \mathrm{nmol} / \mathrm{L}$, and insulin auto-antibody $<1 \mathrm{nmol} / \mathrm{L}$. Throughout the course of the hospital stay, patient experienced drowsiness when her fingerstick glucose was $<50 \mathrm{mg} / \mathrm{dL}$ which was reversed with dextrose 50\% IV. CT abdomen w/pancreatic protocol showed a 28 x $17 \mathrm{~mm}$ mass near uncinate pancreas with $1.4 \mathrm{~cm}$ retroperitoneal lymphadenopathy and two $<1 \mathrm{~cm}$ hepatic liver lesions (Figure 1). Patient was started on diazoxide $100 \mathrm{mg}$ oral (PO) every 8 hours and her fingerstick normalized to above $100 \mathrm{mg} / \mathrm{dL}$. Surgery was recommended for diagnostic and therapeutic treatment of presumed insulinoma, but she refused despite strong recommendations by medical personnel.

The patient was lost to follow up for 5 years and on return had diffuse liver metastases and extensive peripancreatitic lymph nodes extending to the mesentery and encasing the superior mesenteric artery on Magnetic Resonance Imaging (MRI; Figure 2). Computed Tomography (CT)-guided liver biopsy revealed carcinoid-type metastatic neuroendocrine tumors (negative for gastrin/insulin/somatostatin). However, as she clinically had Whipple's triad (hypoglycemia $<50$ $\mathrm{mg} / \mathrm{dL}$, symptoms of hypoglycemia, and reversal after glucose administration) and serum levels consistent with insulinoma, her diagnosis remained metastatic insulinoma. She was then started on monthly octreotide and sunitinib with good symptomatic and clinical responses. However, sunitinib was discontinued due to both non-adherence and dermatologic adverse reactions including pedal edema and petechiae-like lesions, on her lower and upper extremities in the absence of thrombocytopenia. These lesions resolved after stopping sunitinib. Octreotide was continued. Six months later, due to multiple hypoglycemic episodes, repeat MRI of the abdomen/pelvis showed progression of disease with larger and more numerous liver metastases. Everolimus 10mg daily was started.



Figure 1a. The mass to the left lateral aspect of the uncinated process extends along the superior portion of the distal transverse duodenum and adjacent to the superior mesenteric artery measures 28 x $17 \mathrm{~mm}$ maximal cross-sectional diameter. Although this is immediately adjacent to the superior mesenteric artery fat plane, the superior mesenteric artery is maintained 


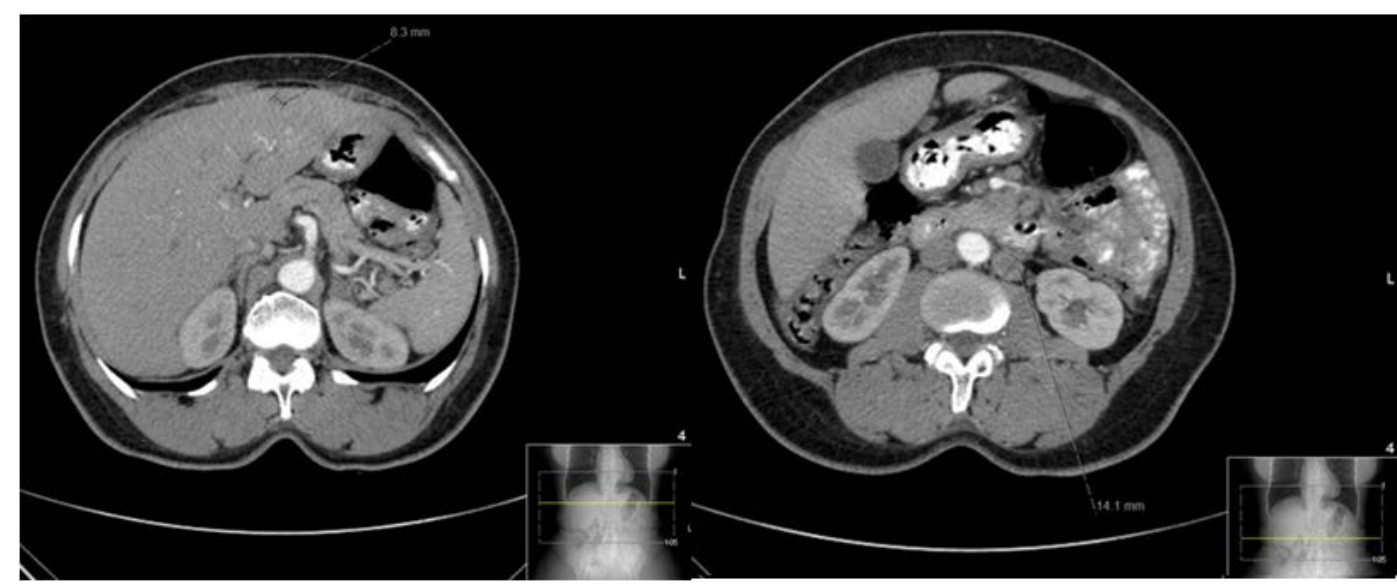

Figure 1b. 6 mm hypodensity in the R hepatic lobe. 1c. Retroperitoneal LAD of $1.4 \mathrm{~cm}$



Figure 2. MRI abdomen w/ and w/o contrast. 2a. T1 isointense signal which may represent the patient's primary neoplasm given history of insulinoma. There is extensive bulky peripancreatic lymphadenopathy which extends along the root of the mesentery and encases the superior mesenteric artery inferior to the pancreas. Bulky and enlarged bilateral renal hilar lymph nodes are present measuring up to $2.9 \mathrm{~cm}$. $2 \mathrm{~b}$ : T2 sequence of coronal section of MRI abdomen/pelvis showing liver metastasis



Figure 3. Histology of Liver metastasis from metastatic insulinoma showing carcinoid-type metastatic neuroendocrine tumors (negative for gastrin/insulin/somatostatin) 


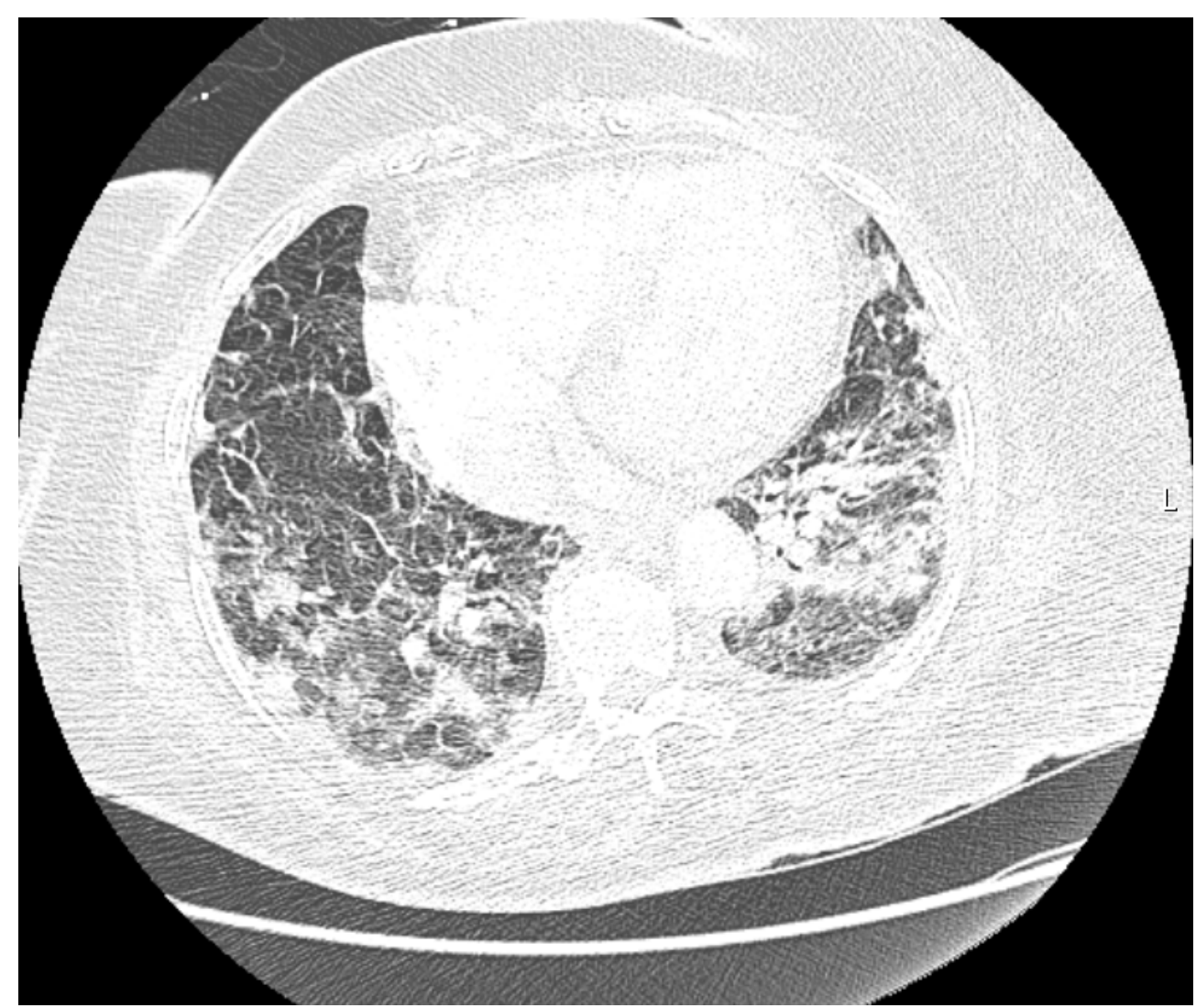

Figure 4. Progression of diffuse, scattered consolidations/nodularity with surrounding ground glass opacifications, which was diagnosed as

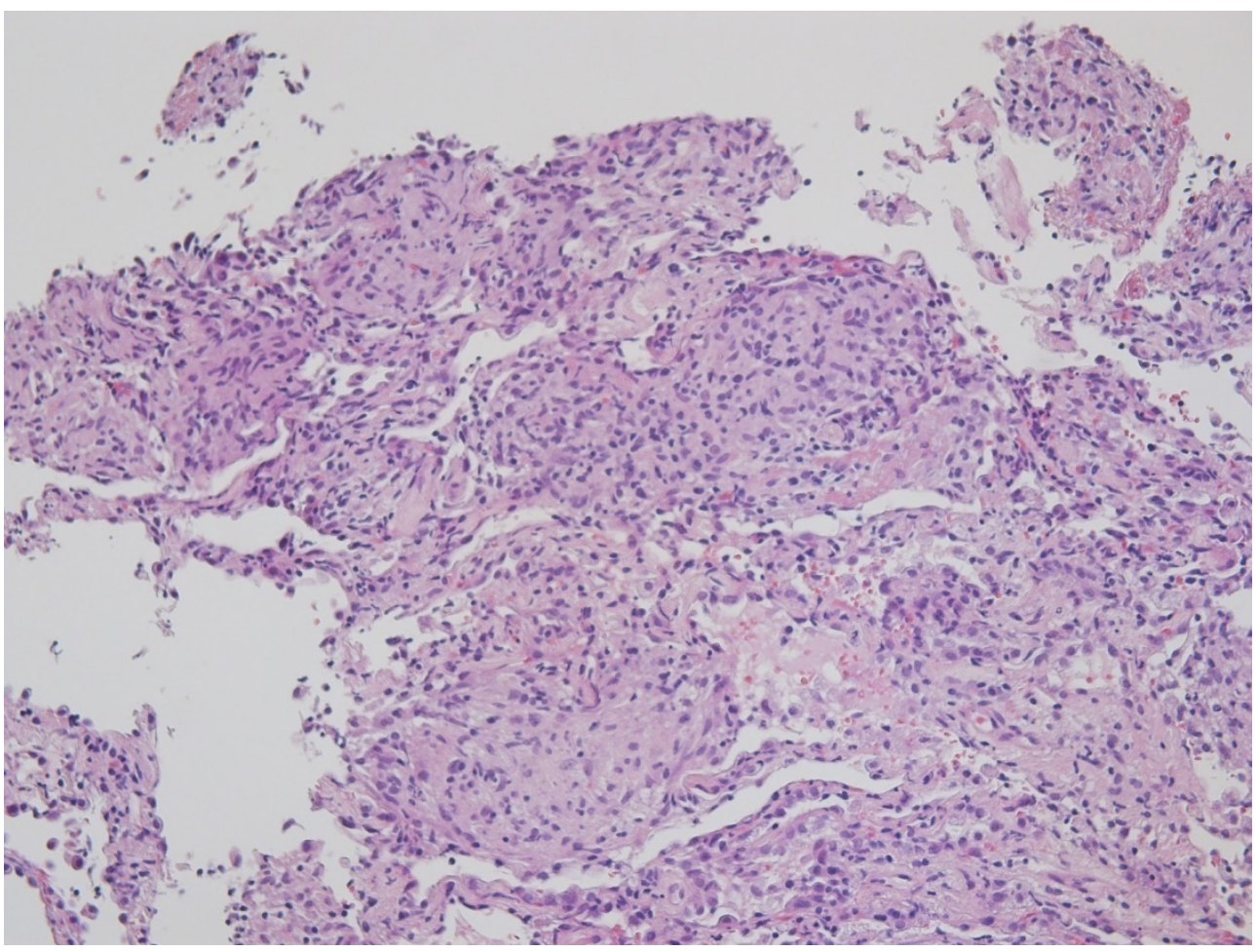

Figure 5. Lung biopsy showing cryptogenic organizing pneumonia, non-necrotizing granulomatous inflammation, and reactive epithelial changes. 20x magnification

On her return to the clinic six months later, the patient was noted to have sudden dyspnea and desaturation to $89 \%$ despite $2 \mathrm{~L} / \mathrm{min}$ of oxygen therapy. Patient underwent CTA which showed no PE, but a progression of diffuse, scattered consolidations/nodularity with surrounding ground glass opacifications (Figure 4). Transbronchial biopsy was done through the left lower lobe and lingula. No malignant cells or infectious agents were seen. On pathological examination, cryptogenic organizing pneumonia, chronic non-necrotizing granulomatous inflammation, and reactive epithelial changes were noted. Prednisone $1 \mathrm{mg} / \mathrm{kg} /$ day for 8 weeks was started for the management 
of cryptogenic organizing pneumonia. Within 48 hours, patient's symptoms and hypoxemia improved to 98-99\% on room air and her repeat CT chest showed that her diffuse scattered consolidations/nodularity with ground glass opacities were markedly decreased from prior CT scans.

Despite repeated admissions to the hospital due to hypoglycemia of 60 or less secondary to patient's non-adherence to diazoxide, she is clinically stable on octreotide, diazoxide, everolimus, and prednisone administrations since her first diagnosis of metastatic insulinoma ten years ago.

\section{Discussion}

\subsection{How to Diagnose Insulinoma}

The standard to diagnose insulinoma is a 72-hour fasting period where blood samples for blood glucose, beta-hydroxybutyrate, C-peptide, Pro-insulin, insulin are collected every 6 hours until glucose is less than $60 \mathrm{mg} / \mathrm{dL}$; at which point, these serum levels are measured every one to two hours. The test concludes when the patient experiences Whipple's triad with a glucose $<55 \mathrm{mg} / \mathrm{dL}$; when patient's plasma glucose concentration is $\leq 45 \mathrm{mg} / \mathrm{dL}$; when the patient has symptoms or signs of hypoglycemia; or, when 72 hours have elapsed [1]. In contrast, Hirshberg of the National Institutes for Health published a study in 2000 that proposed a 48-hour fasting period. At the end of 48 hours, $95 \%$ of 127 patients with insulinoma were detected [2]. However, in more recent work, Service et al have noted that a series of 205 patients with insulinoma, $14 \%$ of patients did not develop symptoms until after 48 hours of fasting, bringing into question the generalizability of the 48-hour fasting period as a diagnostic tool [3].

The values of the serum measurements include plasma insulin concentrations of at least $3 \mu \mathrm{U} / \mathrm{ml}$ (18 pmol/liter), plasma C-peptide concentrations of at least $0.6 \mathrm{ng} / \mathrm{ml}$ (0.2 nmol/liter), and plasma proinsulin concentrations of at least $5.0 \mathrm{pmol} /$ liter when the fasting plasma glucose concentrations are below $55 \mathrm{mg} / \mathrm{dl}$ (3.0 mmol/liter) [4]. Once these measurements are made, levels of hypoglycemic agents (e.g. sulfonylureas) and antibodies to insulin are also tested to rule out surreptitious, malicious use of medication and immunologic processes.

Once the diagnostic serum levels are measured, diagnostic imaging modalities, including CT (Sensitivity 70-80\%), MRI (sensitivity 85\%), endoscopic pancreatic ultrasonography (sensitivity 90\%), somatostatin receptor-based imaging (i.e. 88Ga-dotate PET/CT [preferred] with sensitivity of $97 \%$ or somatostatin receptor scintigraphy with sensitivity of $60 \%$; reference [5]) are used to locate the tumor. However, due to insulinomas' small size (often $<1.0 \mathrm{~cm}$ in diameter), imaging may not be able to locate a mass. Thus, a more invasive hepatic insulin levels are measured after selective pancreatic arterial calcium injections are made if the imaging modalities are equivocal [6].

In our patient, she underwent a fasting period which was terminated before 72 hours with a serum glucose level $<45$. Her serum insulin level was greater than $3 \mathrm{mcU} / \mathrm{mL}$ at $18.77 \mathrm{mcU} / \mathrm{mL}$; her C-peptide was greater than $0.6 \mathrm{pmol} / \mathrm{L}$ at $3.85 \mathrm{nmol} / \mathrm{L}$; and her insulin auto-antibody level was undetectable at $<1 \mathrm{nmol} / \mathrm{L}$. Both $\mathrm{CT}$ with contrast, MRI of abdomen/pelvis were suggestive of metastases to the retroperitoneal space, liver, mesentery, and perimesenteric artery. Due to the limited availability of specialized tests including somatostatin receptor-based imaging or invasive hepatic insulin level measurement after selective pancreatic arterial calcium injections, they were not performed to localize the disease as patient already had metastatic disease. Although the patient's CTguided biopsy of a presumed metastatic liver lesion showed a carcinoid-type lesion that was negative for insulin/gastrin/somatostatin, patient's clinical and serum levels were diagnostic for insulinoma. We believe that this biopsy was performed on a non-functional liver lesion of a metastatic insulinoma.

What follows is a review of treatment options of insulinoma.

\subsection{Treatments}

\subsubsection{Surgical Management}

The initial and definitive treatment for insulinoma has traditionally been open surgical management, based on a retrospective study of a 60-year period of 224 patients at Mayo Clinic [1]. A recent review and a meta-analysis advocates that laparoscopic resection is associated with improved outcomes, having decreased mortality and length of stay $[7,8]$. Benign, solitary tumors require surgical excision. Multiple insulinomas require local excision of any tumors found in pancreatic head and a distal subtotal pancreatectomy. Patients with persistent hypoglycemia after surgery are recommended for repeat operation. For potentially resectable hepatic-isolated metastasis, current recommendations are to excise the metastasis along with primary tumor. Several case studies studying endoscopic ultrasound and intraoperative ultrasound guided ethanol ablation has shown some success in poor surgical candidates for small localized lesions, however several of these procedures have developed complications ranging from bleeding to pseudocyst formation and pancreatitis $[9,10,11]$. Other strategies which can be used for particularly hepatic predominant metastasis include hepatic artery embolization, radiofrequency ablation, and cryoablation.

\subsection{Medical Therapies}

\subsubsection{Diazoxide}

For insulinomas that are unable to be resected, the initial medical management of hypoglycemia is with the use of diazoxide, which is an antihypertensive, antidiuretic benzothiadiazine derivative. In the presence of magnesium and ATP, diazoxide opens pancreatic beta-cell potassiumATP channel and hyperpolarizes the beta-cell-inhibiting insulin release [12]. The dose of diazoxide is $150-200 \mathrm{mg}$ in two or three divided doses per day and titrated to a maximum dose of $400 \mathrm{mg} /$ day [13]. In $47 \%$ of $40 \mathrm{UK}$ patients with insulinoma, side effects included fluid retention and hirsutism which were well-tolerated [14]. 


\subsubsection{Somatostatin Analogs: Octreotide, Lanreotide}

For patients with hypoglycemia refractory to diazoxide, octreotide is recommended. However, it is to be used with caution in insulinoma due to paradoxical transient worsening of hypoglycemia, presumably attributed to glucagon secretion inhibition and with some relation to expressed tumor somatostatin receptors [15]. Several small studies have shown somatostatin analogs to be associated with tumor and metabolic regression when associated with SSTR2 immunoreactivity $[16,17]$. Lanreotide also has a similar efficacy profile to octreotide for neuroendocrine tumors and is available as a long acting depot $[18,19,20]$.

\subsubsection{Molecularly Targeted Therapy: Everolimus, Bevacizumab, Sunitinib}

For refractory cases, molecularly targeted therapy appears to be a favorable option particularly for advanced disease or rapid metastasis. Pancreatic neuroendocrine tumors are known to overexpress Insulin Growth Factor-1 (IGF-1) which stimulates mammalian target of rapamycin (mTOR). Inhibitors of this molecule, everolimus and sirolimus, have been studied in solid organ tumors and their mechanisms of action are two-fold: arresting tumor growth at G1/S cell cycle and inhibition of angiogenesis [21]. In a Phase III trial (RADIANT-3) published in 2011, 410 patients with advanced, low-grade or intermediate-grade (unresectable or metastatic) pancreatic neuroendocrine tumors with radiologic progression were separated to a treatment arm of everolimus (10 mg once daily; 207 patients), and a placebo group of 203 patients. Some of these patients had metastatic insulinoma. Median progression free survival (PFS) was 11 months with everolimus, compared with 4.6 months with placebo (HR=0.35; 95\% CI 0.27-0.45; P <0.001). 64\% of the treatment group experienced some degree of tumor shrinkage, as compared with $21 \%$ in the placebo group. Drug-related adverse events included stomatitis (64\% of treatment group vs. $17 \%$ of placebo), rash (49\% vs. $10 \%)$, diarrhea (34\% vs. 10\%), fatigue (31\% vs. 14\%), infections (23\% vs. 6\%), which were primarily upper respiratory, anemia (6\% vs. $0 \%$ ) and hyperglycemia (5\% vs. $2 \%$ ). Dose adjustments were made in some of these patients and everolimus had to be discontinued in $13 \%$ of patients due to pneumonitis, fatigue, and interstitial lung disease. Of note, the death of one patient with insulinoma in the everolimus group, despite glucocorticoid therapy, was considered to be everolimus-related acute respiratory distress syndrome. However, it was unclear whether this patient exhibited features of pneumonitis or cryptogenic organizing pneumonia (COP) [22].

\subsubsection{Bevacizumab}

The use of bevacizumab, a direct vascular endothelial growth factor antibody, was studied in a randomized phase II trial of 150 patients with metastatic well-differentiated pancreatic neuroendocrine tumors. The treatment group consisted of bevacizumab (10 mg/kg IV q 2 weeks) and everolimus (10 mg po qd) vs Everolimus alone. Both groups received concurrent standard dose octreotide (20 mg IM). The B+E showed a superior progression free survival (16.7 months vs 14 months in $\mathrm{E}$ alone, $\mathrm{HR}=0.80$; $95 \%$ CI: $0.55,1.17)$ and response rates (31\% vs $12 \%$ in $\mathrm{E}$ alone). However, patients experienced higher frequency of adverse events in $\mathrm{B}+\mathrm{E}$ group including diarrhea, hyponatremia, hypophosphatemia, proteinuria, hypertension, and pneumonitis (12.33\% in $\mathrm{B}+\mathrm{E}$ vs $8.11 \%$ in $\mathrm{E}$ only) [27]. In another ongoing Phase II trial, 22 patients received bevacizumab with octreotide administration compared to placebo. They noted a PFS rates of 95\% and $54 \%$ in 6- and 12-month period with minimal systemic toxicity of no grade 3-4 Adverse events except 36\% of grade 3 hypertension [28].

\subsubsection{Sunitinib}

Sunitinib, a multi-targeted TK inhibitor, notably with VEGF-inhibition properties, has shown to have some ability in prolonging periods of progression free survival in a phase II trial of 109 patients with advanced neuroendocrine tumors and a phase III trial of 171 patients with progressing pancreatic neuroendocrine tumors. Compared to placebo, it showed increases in PFS, overall survival (OS), and objective response rates [29,30]. In a case report, sunitinib was shown to successfully treat metastatic insulinoma, however, the patient had multiple events of symptomatic hypoglycemia. The patient could tolerate the regimen after adjusting dosage and diet [31]. In comparing RADIANT-3 and a study from Raymond et al, sunitinib showed slightly higher progression free survival vs everolimus (11.4 months vs 11 months) and increased tumor response rate (9\% vs 5\%) [22,30].

\subsubsection{Chemotherapy: Streptozocin, Dacarbazine, Temozoloamide; Gemcitabine and Oxaliplatin}

Molecular targeted therapy is now preferable as standard cytotoxic medication has limited efficacy, particularly in advanced insulinomas [31]. Furthermore, the adverse effects of chemotherapy have further hindered its acceptance as first line therapy. As per Demirkan et al, well-differentiated neuroendocrine tumors do not show high sensitivity to chemotherapy including streptozocin with 5-fluorouracil or daunorubicin because of their low mitotic rates, high levels of antiapoptotic protein bcl-2 and increased expression of the multi-drug resistant gene. However, monoagents or combination therapy of temozolomide, capecitabine and oxaliplatin showed efficacy in phase II trials [32].

\subsubsection{Cryptogenic Pneumonia in other Diseases}

As there is very limited number of metastatic insulinoma undergoing treatment with mTOR inhibitors, other diseases with the use of everolimus were researched. Progressive interstitial pneumonitis had been associated with the use of mTOR inhibitors in patients with advanced renal cell carcinoma and in patients who received renal transplants [23,24,25,26]. The pathophysiology behind mTOR inhibitor-mediated damage to alveoli and small airways is not well understood and remains purely speculative: inflammatory changes to bronchioles and surrounding tissue result in the development of a non-infectious pneumonia that presents initially with cough and dyspnea, but systemic symptoms such as fever can also be noted $[25,26]$. Different treatment methods have been compared, including decreasing the dose of everolimus/sirolimus, stopping mTOR inhibitor therapy, and, combinations of decreasing or stopping mTOR therapy with initiation of corticosteroids. Multiple studies 
showed that over half of patient's affected with pneumonitis were controlled and even had complete resolution with decreasing mTOR inhibitor dose and/or using corticosteroids [23,24,25,26]. However, there also exists another report of a 61-year-old man that was treated with everolimus for metastatic renal carcinoma to the lungs, bones, and left adrenal gland that despite discontinuing everolimus and initiation of corticosteroids, patient died from drug-induced pulmonary toxicity [33].

\section{Conclusion}

In our patient, the diagnosis of everolimus-induced COP in a patient with metastatic insulinoma can be inferred: patient did not experience dyspnea and hypoxemia prior to the administration of everolimus; patient's CT scan did not demonstrate a pattern of COP prior to the administration; patient's symptoms and CT scan improved with a corticosteroids that combat against a speculative pathophysiology of COP, which is an inflammatory changes to bronchioles and surrounding tissue; and, there are other reports suggestive of an association of everolimus and COP as discussed above. According to the Naranjo probability scale, the association between everolimus and COP in a patient with metastatic insulinoma was a score of 5 , which suggests a probable association.

Insulinoma is a rare cause of a common presentation of hypoglycemia. Although insulinomas, when detected, have a favorable mortality rate after a surgical removal, there are some patients who present with inoperable disease. In our patient, everolimus is a valid treatment option for metastatic insulinoma demonstrated by decreased numbers of emergency room visits and decreased episodes of hypoglycemia. Although the incidence of insulinomas and pulmonary complications are rare, the medical community should be aware of fatal complications and treatments of rare diseases. While pulmonary complications have been cited as reasons for discontinuation of everolimus therapy (34), our case report highlights the use of steroids as a viable therapeutic strategy that allows successful therapy with everolimus to be continued.

In conclusion, prednisone administration is a viable treatment option for cryptogenic organizing pneumonia which was most likely caused by the administration of everolimus for the treatment of metastatic insulinoma.

\section{Acknowledgements}

This work is supported, in part, by the efforts of Dr. Moro O. Salifu M.D., M.P.H., M.B.A., M.A.C.P., Professor and Chairman of Medicine through NIH Grant number S21MD012474.

\section{References}

[1] Service FJ, McMahon MM, O’Brien PC, Ballard DJ. Functioning insulinoma - incidence, recurrence, and long-term survival of patients: a 60-year study. Mayo Clin Proc. 1991; 66: 711-719.
[2] Hirshberg B, Livi A, Bartlett DL, et al. Forty-eight-hour fast: the diagnostic test for insulinoma. J Clin Endocrinol Metab 2000; 85: 3222-6.

[3] Service, F. J., \& Natt, N. (2000). The Prolonged Fast. The Journal of Clinical Endocrinology \& Metabolism, 85(11), 3973-3974.

[4] Service FJ. Hypoglycemic disorders. N Engl J Med. 1995; 332(17) 1144-52.

[5] Gabriel M, Decristoforo C, Kendler D, et al. 68Ga-DOTA-Tyr3octreotide PET in neuroendocrine tumors: comparison with somatostatin receptor scintigraphy and CT. J Nucl Med. 2007; 48: 508-518.

[6] Grant CS. Insulinoma. Best Pract Res Clin Gastroenterol. 2005; 19 783-798.

[7] Su, A., Ke, N., Zhang, Y., Liu, X., Hu, W., Tian, B., \& Zhang, Z. (2014). Is laparoscopic approach for pancreatic insulinomas safe? Results of a systematic review and meta-analysis. Journal of Surgical Research, 186(1), 126-134.

[8] Mehrabi A, Fischer L, Hafezi M, et al. A systematic review of localization, surgical treatment options, and outcome of insulinoma. Pancreas 2014; 43: 675-86.

[9] Levy, M., \& Topazian, M. (2011). Sp694 Ultrasound Guided Ethanol Ablation of Insulinomas: A New Treatment Option. Gastrointestinal Endoscopy, 73(4).

[10] Jürgensen C., Schuppan D., Neser F., Ernstberger J., Junghans U., Stölzel U. EUS-guided alcohol ablation of an insulinoma. Gastrointestinal Endoscopy. 2006; 63(7): 1059-1062.

[11] Deprez P. H., Claessens A., Borbath I., Gigot J. F., Maiter D. Successful endoscopic ultrasound-guided ethanol ablation of a sporadic insulinoma. Acta Gastroenterologica Belgica. 2008; 71: 333-337.

[12] Doyle, M.E. Pharmacological agents that directly modulate insulin secretion. Pharmacol. Rev. 2003, 55, 105-131.

[13] Shin JJ, Gorden P, Libutti SK. Insulinoma: pathophysiology, localization and management. Future Oncol. 2010; 6: 229-237.

[14] Gill GV, Rauf O, MacFarlane IA. Diazoxide treatment for insulinoma: a national UK survey. Postgrad Med J. 1997; 73(864): 640-641.

[15] Plöckinger U, Wiedenmann B. Neuroendocrine tumors. Biotherapy. Best Pract Res Clin Endocrinol Metab 2007; 21: 145-62.

[16] Romeo S, Milione M, Gatti A, Fallarino M, Corleto V, Morano S, et al. Complete clinical remission and disappearance of liver metastases after treatment with somatostatin analogue in a 40-year-old woman with a malignant insulinoma positive for somatostatin receptors type 2. Horm Res. 2006;65(3):120-5.

[17] von Eyben FE, Grodum E, Gjessing HJ, Hagen C, Nielsen H. Metabolic remission with octreotide in patients with insulinoma. $\mathrm{J}$ Intern Med (1994) 235: 245-810.

[18] Eriksson B., Renstrup J., Imam H., Öberg K. High-dose treatment with lanreotide of patients with advanced neuroendocrine gastrointestinal tumors: Clinical and biological effects. Annals of Oncology. 1997; 8(10): 1041-1044.

[19] Tomassetti P, Migliori M, Gullo L. Slow - release lanreotide treatment in endocrine gastrointestinal tumors. Am J Gastroenterol 1998; 93: 1468-1471.

[20] Toumpanakis C, Caplin ME. Update on the role of somatostatin analogs for the treatment of patients with gastroenteropancreatic neuroendocrine tumors. Semin Oncol. 2013;40:56-68.

[21] Lin, T., Leung, C., Nguyen, K. T., \& Figlin, R. A. (2016). Mammalian target of rapamycin (mTOR) inhibitors in solid tumours. Clinical Pharmacist. Retrieved August 1, 2018, from https://www.pharmaceutical-journal.com/research/reviewarticle/mammalian-target-of-rapamycin-mtor-inhibitors-in-solidtumours/20200813.article?firstPass=false\#comments_form.

[22] Yao JC, Shah MH, Ito T, Bohas CL, Wolin EM, Van Cutsem E, et al. Everolimus for advanced pancreatic neuroendocrine tumors. N Engl J Med. 2011; 364(6): 514-523.

[23] Weiner SM, Sellin L, Vonend O, et al. Pneumonitis associated with sirolimus: clinical characteristics, risk factors and outcome--a single-centre experience and review of the literature. Nephrol Dial Transplant 2007; 22: 3631.

[24] Champion L, Stern M, Israël-Biet D, et al. Brief communication: sirolimus-associated pneumonitis: 24 cases in renal transplant recipients. Ann Intern Med 2006; 144: 505.

[25] Pham PT, Pham PC, Danovitch GM, et al. Sirolimus-associated pulmonary toxicity. Transplantation 2004; 77: 1215. 
[26] White DA, Camus P, Endo M, et al. Noninfectious pneumonitis after everolimus therapy for advanced renal cell carcinoma. Am J Respir Crit Care Med 2010; 182:396-403.

[27] Randomized phase II study of everolimus (E) versus everolimus plus bevacizumab $(\mathrm{E}+\mathrm{B})$ in patients $(\mathrm{Pts})$ with locally advanced or metastatic pancreatic neuroendocrine tumors (pNET), CALGB 80701 (Alliance). Matthew H. Kulke, Donna Niedzwiecki, Nathan R. Foster, Briant Fruth, Pamela L. Kunz, Hagen F. Kennecke, Edward M. Wolin, and Alan P. Venook. Journal of Clinical Oncology 2015 33: 15_suppl, 4005-4005.

[28] Multicenter prospective phase II trial of bevacizumab (bev) for progressive pancreatic neuroendocrine tumor (PNET). Timothy J. Hobday, Jun Yin, Adam Pettinger, Jonathan R. Strosberg, Diane Lauren Reidy, Helen X. Chen, and Charles Erlichman. Journal of Clinical Oncology 2015 33: 15_suppl, 4096-4096.

[29] Kulke MH, Lenz HJ, Meropol NJ, et al. Activity of sunitinib in patients with advanced neuroendocrine tumors. J Clin Oncol 2008; 26: 3403-10.
[30] Raymond E, Dahan L, Raoul JL, Bang YJ, Borbath I, LombardBohas C, et al. Sunitinib malate for the treatment of pancreatic neuroendocrine tumors. N Engl J Med. 2011; 364: 501-13.

[31] Chen J, Wang C, Han J, et al. Therapeutic effect of sunitinib malate and its influence on blood glucose concentrations in a patient with metastatic insulinoma. Expert Rev Anticancer Ther. 2013; 13: 737-743.

[32] Demirkan B, Eriksson B. Systemic treatment of neuroendocrine tumors with hepatic metastases. Turk J. Gastroenterol. 2012; 23(5): 427-37.

[33] Nazer L, Alnajjar T, Salah S, Khzouz J, Alfaqeer N, Qandeel M. Fatal case of cryptogenic organizing pneumonia associated with everolimus. Ann Saudi Med. 2014; 34(5): 437-439.

[34] Bernard V, Lombard-Bohas C, Taquet MC, et al. Efficacy of everolimus in patients with metastatic insulinoma and refractory hypoglycemia. Eur J Endocrinol 2013; 168: 665-674. 